\title{
RXTE broadband X-ray spectra of intermediate polars and white dwarf mass estimates
}

\author{
V. Suleimanov ${ }^{1,2,4}$, M. Revnivtsev ${ }^{2,3}$, and H. Ritter ${ }^{2}$ \\ 1 Kazan State University, Kremlevskaya str. 18, 420008 Kazan, Russia \\ e-mail: vals@ksu.ru \\ 2 Max-Planck-Institut für Astrophysik, Karl-Schwarzschild-Str. 1, 85741 Garching bei München, Germany \\ 3 Space Research Institute, Russian Academy of Sciences, Profsoyuznaya 84/32, 117810 Moscow, Russia \\ 4 Astronomy Division, PO Box 3000, 90014 University of Oulu, Finland
}

Received 13 May 2004 / Accepted 15 December 2004

\begin{abstract}
We present results of an analysis of broadband X-ray spectra of 14 intermediate polars obtained with the $R X T E$ observatory (PCA and HEXTE spectrometers, 3-100 keV). For this we have calculated theoretical models of the structure and the emergent spectrum of the post-shock region of intermediate polars. By fitting theoretical model spectra to the observed spectra we derive estimates for the masses of the white dwarfs. We compare the resulting masses with masses obtained by other authors and other methods. The masses obtained by us are smaller than the masses obtained by using PCA and GINGA data, and they are in good agreement with the masses derived from radial velocity studies.
\end{abstract}

Key words. stars: binaries: close - stars: binaries: spectroscopic - X-ray: binaries - stars: novae, cataclysmic variables

\section{Introduction}

Intermediate polars (IP) form a sub-class of magnetic cataclysmic variables (mCV) (Warner 1995). A white dwarf (WD) accretes matter from a companion star (typically a red dwarf) that fills its Roche lobe. This matter forms an accretion disc inside the Roche lobe of the WD. The disc, however, is disrupted by the WD magnetic field at some distance from the WD surface. As a result the accreting matter freely falls on to the WD surface and forms a strong shock near its surface. The post-shock matter has a high temperature $(\sim 10-20 \mathrm{keV})$ and emits X-rays via optical thin thermal plasma emission (Lamb \& Masters 1979).

The temperature of the post-shock matter depends on the WD mass and, therefore, the X-ray spectra of IPs can be used for WD mass determination (Rothschild et al. 1981). Ishida (1991) investigated IPs and polars by using GINGA/LAC observations. He fitted the X-ray spectra with single temperature bremsstrahlung models. Then WD masses were estimated by equaling the resulting best fit bremsstrahlung temperature to the maximal shock temperature. However, a significant part of the post shock region has cooler temperatures due to radiative cooling and, as mentioned by Ishida, WD masses obtained in this way should be considered as lower limits only.

In order to estimate the masses of WDs more precisely using X-ray spectra of IPs one needs to calculate the temperature and emissivity distribution in the post-shock region (PSR). The structure of the PSR was investigated by Aizu (1973), Wu et al. (1994), Woelk \& Beuermann (1996),
Cropper et al. (1999), see also Frank et al. (2002) and Wu (2000). Wu et al. (1994) and Woelk \& Beuermann (1996) took into account cyclotron cooling, which can be important for polars. In these papers it was demonstrated that cyclotron cooling is not important for $\mathrm{mCV}_{\mathrm{s}}$ with surface magnetic fields less than 10 MG. The model of Wu et al. (1994) was used by Cropper et al. (1998, 1999) and Ramsay (2000) for the fitting of the IPs spectra observed by GINGA/LAC and RXTE/PCA respectively. Beardmore et al. (2000) used a simple analytical model of the PSR (see Frank et al. 2002) to analyse the spectra of V1223 Sgr observed by GINGA/LAC and ASCA. Ezucka and Ishida (1999) estimated the mass of the WD of nine IPs using emission lines ratios from ASCA observations.

The main systematic uncertainty in these works is caused by the fact that neither the GINGA/LAC nor the RXTE/PCA detectors provide information at energies $\gtrsim 20 \mathrm{keV}$. Therefore, it is hard to get solid estimates for the masses of the WDs if the mass is $>0.6 M_{\odot}$, because in that case the maximal post-shock temperature is $>20 \mathrm{keV}$.

Moreover, the X-ray continuum of IPs in the range $<20 \mathrm{keV}$ is modified by interstellar and intrinsic absorption, and by a possible reflection component. The intrinsic absorption is not yet well understood due to the possible inhomogeneous and complex geometry of the accretion flow (Done \& Magdziarz 1998). Usually simple models, such as a partial covering by a cold and a partially ionized absorber are used (see Croper et al. 1999; Beardmore et al. 2000). It is also clear that hard X-rays from the PSR are reflected by Compton scattering from the 
white dwarf surface which results in an additional continuum component (see Beardmore et al. 2000; Cropper et al. 1998). Therefore, it is difficult to determine the correct intrinsic X-ray continuum of the PSR from the observed spectra of IPs at energies less 20-25 keV and hence to derive reliable estimates for the white dwarf masses. As a result, the white dwarf masses obtained from X-ray continuum fitting are very often inconsistent with masses derived by other methods, e.g. from radial velocity studies (Schwope et al. 2002). All of the above-mentioned problems are less important if spectra which include the harder $\mathrm{X}$-ray energy band (up to $100 \mathrm{keV}$ ), for example the total energy band of the RXTE observatory spectrometers $(3-250 \mathrm{keV})$ are used.

On the other hand, WD masses derived from radial velocity studies are often not very reliable because the orbital inclination of a system is well known for eclipsing systems only. If the inclination angle is unknown, additional assumptions have to be made. In most cases this is done by prescribing a mass radius relation for the secondary which, in turn, is equivalent to assuming a secondary mass orbital period relation. However, the use of such assumptions can introduce a significant bias; for example, Belle et al. (2003) have obtained a mass of the WD in EX Hya which is two times larger than that obtained by Beuermann et al. 2003. While Belle et al. (2003) used a main sequence mass radius relation, Beuermann et al. (2003) determined the inclination from X-ray eclipse data. Therefore, it is necessary to compare the WD masses obtained by using X-ray data with WD masses obtained from optical methods. If both methods yield the same result, then this gives us more confidence in the resulting mass.

In this paper we present results of WD mass estimates for 14 bright IPs, obtained from broad-band (3-100 keV) RXTE spectra. Most of the analysed sources have a statistically significant signal at energies $>10 \mathrm{keV}$. The observed spectra have been fitted with PSR model spectra. Details of the model are described in Sect. 2. We compare the WD masses resulting from our best fit parameters with WD masses that were obtained by other authors from X-ray data and by optical methods.

\section{The model}

The structure of the stationary post-shock region in a planeparallel one-dimensional geometry is described (see e.g. Cropper et al. 1999) by the mass continuity equation

$\frac{\mathrm{d}}{\mathrm{d} z}(\rho v)=0$,

the momentum equation

$\frac{\mathrm{d}}{\mathrm{d} z}\left(\rho v^{2}+P\right)=-\frac{G M_{\mathrm{wd}}}{z^{2}} \rho$,

the energy equation

$v \frac{\mathrm{d} P}{\mathrm{~d} z}+\gamma P \frac{\mathrm{d} v}{\mathrm{~d} z}=-(\gamma-1) \Lambda$,

and the ideal-gas law

$P=\frac{\rho k T}{\mu m_{\mathrm{H}}}$.

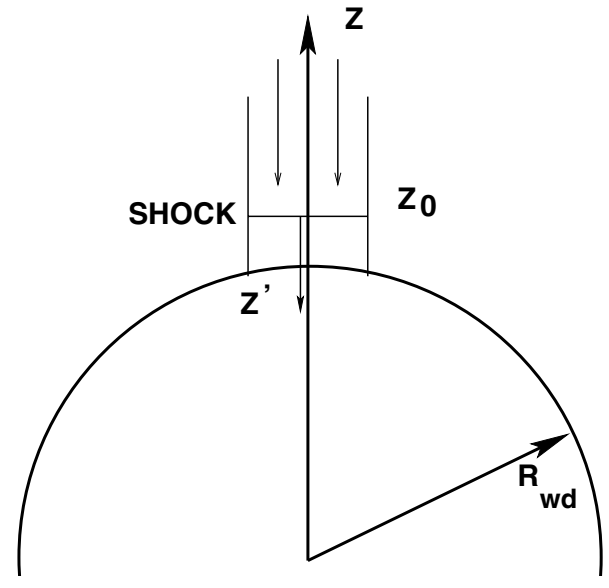

Fig. 1. Geometry of the post-shock region model.

Here $z$ is the spatial coordinate (see Fig. 1), $v$ the velocity of matter, $\rho$ the density, $T$ the temperature, $P$ the gas pressure, $\gamma=5 / 3$ the adiabatic index, and $\mu=0.62$ the mean molecular weight. The cooling rate $\Lambda$ due to thermal optically thin radiation is given by:

$\Lambda=\left(\frac{\rho}{\mu m_{\mathrm{H}}}\right)^{2} \Lambda_{\mathrm{N}}(T)$,

where $\Lambda_{\mathrm{N}}(T)$ is the cooling function. In our work $\Lambda_{\mathrm{N}}(T)$ is taken for solar chemical composition as calculated and tabulated by Sutherland \& Dopita (1993).

Equation (1) has the integral

$\rho v=a$,

where $a$ is the local mass accretion rate per unit area. The local mass accretion rate is a free parameter of the model. However, we found that the spectrum of the emergent radiation is rather insensitive to this parameter within reasonable limits, (i.e. as long as the emission region is optically thin with respect to free-free scattering).

Equations (2) and (3) can be rewritten using Eq. (6) with the substitution $z^{\prime}=z_{0}-z$, where $z_{0}$ is the shock coordinate (see Fig. 1):

$\frac{\mathrm{d} v}{\mathrm{~d} z^{\prime}}=g\left(z^{\prime}\right) \frac{1}{v}-\frac{1}{a} \frac{\mathrm{d} P}{\mathrm{~d} z^{\prime}}$,

$\frac{\mathrm{d} P}{\mathrm{~d} z^{\prime}}=\frac{(\gamma-1) \Lambda a+g\left(z^{\prime}\right) \gamma P a / v}{\gamma P-a v}$

where

$g\left(z^{\prime}\right)=\frac{G M_{\mathrm{wd}}}{\left(z_{0}-z^{\prime}\right)^{2}}$.

Equations (7) and (8) were solved by the shooting method from $z=z_{0}\left(z^{\prime}=0\right)$ to $z=R_{\mathrm{wd}}\left(z^{\prime}=z_{0}-R_{\mathrm{wd}}\right)$ with the following boundary conditions at $z=z_{0}$ :

$v_{0}=0.25 \sqrt{2 G M_{\mathrm{wd}} / z_{0}}$,

$\rho_{0}=\frac{a}{v_{0}}$, 


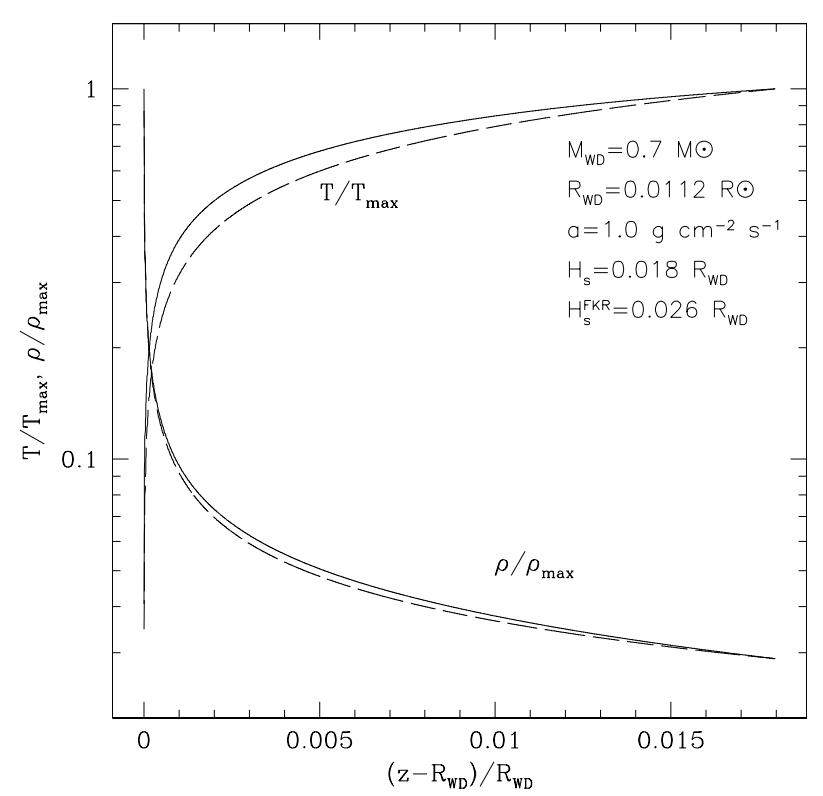

Fig. 2. Temperature and density profiles for one of the PSR models. The dashed lines show a simple analytic solution of the structure of the post shock region (Frank et al. 2002).

$P_{0}=3 a v_{0}$,

$T_{0}=3 \frac{\mu m_{\mathrm{H}}}{k} v_{0}^{2}$.

The shock position $z_{0}$ was found iteratively from the additional boundary condition: $v=0$ at the WD surface. The WD radius was calculated from the Nauenberg (1972) WD mass-radius relation:

$R_{\mathrm{wd}}=7.8 \times 10^{8} \mathrm{~cm}\left[\left(\frac{1.44 M_{\odot}}{M_{\mathrm{wd}}}\right)^{2 / 3}-\left(\frac{M_{\mathrm{wd}}}{1.44 M_{\odot}}\right)^{2 / 3}\right]^{1 / 2}$.

In Fig. 2 we present the temperature and the density profiles of the post shock region resulting from our model for a particular set of boundary values. For comparison we show the profiles obtained by the simple analytical model (Frank et al. 2002):

$T(z)=T_{0}\left(\frac{z-R_{\mathrm{wd}}}{z_{0}-R_{\mathrm{wd}}}\right)^{2 / 5}$,

$\rho(z)=\rho_{0}\left(\frac{z-R_{\mathrm{wd}}}{z_{0}-R_{\mathrm{wd}}}\right)^{-2 / 5}$.

This model is based on the assumption of constant pressure in the post shock region. In our model the pressure grows towards the WD surface. Therefore, in our model the temperature and the density are larger.

In previous investigations (Cropper et al. 1998, 1999; Ramsay 2000; Beardmore et al. 2000) the MEKAL optically thin plasma code (Mewe et al. 1995) was used to calculate both the local continuum and the line emission. But most of the emission lines have energies less than $3 \mathrm{keV}$ (with the exception of a line blend at 6-7 keV). Therefore, most of the emission lines are outside the spectral band $(3-100 \mathrm{keV})$ considered by us.
As the $R X T E / \mathrm{PCA}$ detectors have a relatively poor spectral resolution and have adequate sensitivity only at energies higher than $3 \mathrm{keV}$ we limited ourselves to the study of the continuum emission only. The emergent blend of lines at energies 6-7 keV in the resulting spectrum was mimicked by a broad Gaussian line. In the subsequent fitting of the observed spectra, the center and the width of that line were considered as free parameters.

The model of the emergent spectrum is calculated by summing the local bremsstrahlung spectra:

$F_{\mathrm{E}}=\int_{R_{\mathrm{wd}}}^{z_{0}} j(z) \mathrm{d} z$

where the local spectra were taken in the following form (Zombeck 1990):

$$
\begin{aligned}
& j(z)=9.52 \times 10^{-38}\left(\frac{\rho(z)}{\mu m_{\mathrm{H}}}\right)^{2} T^{-1 / 2}(z)\left(\frac{E}{k T(z)}\right)^{-0.4}
\end{aligned}
$$

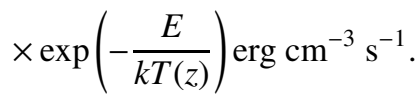

\section{Observations and data reduction}

In order to compare the results of our model calculations with observations we have chosen data of the RXTE observatory that cover a relatively broad energy band (3-250 keV), where IPs release most of their emission. We limited ourselves only to relatively bright IPs, as we are interested in the hard X-ray $(>20 \mathrm{keV})$ part of the spectra of IPs. The list of IPs consists of V1223 Sgr, FO Aqr, EX Hya, AO Psc, TV Col, GK Per, V709 Cas, PQ Gem, V2400 Oph, BG CMi, V405 Aur, V1062 Tau, DO Dra, and TX Col (see Table 1).

The $R X T E / \mathrm{PCA}$ and $R X T E / \mathrm{HEXTE}$ data were reduced by means of standard tasks of the LHEASOFT/FTOOLS 5.2 package. The background subtraction of the PCA detectors was done with the CM17_240 model. The subtraction of the background of the HEXTE detectors is especially important in our case which concentrates on the hard X-ray part of the spectrum of IPs. In order to monitor the background of the detectors, HEXTE rocks by $\pm 1.5^{\circ}$ with respect to the target. We analysed the HEXTE spectra of IPs only if the background spectra obtained at the $+1.5^{\circ}$ and the $-1.5^{\circ}$ offset positions were statistically compatible, in other words, if there were no contaminating sources in the vicinity of our targets. The accuracy of the background subtraction achieved with this method is shown to be at a level of $\sim 0.5-1 \%$ (Rothschild et al. 1998). Practically for all the sources studied, the systematic uncertainties of the background subtraction are not important.

\section{Results}

The X-ray spectra of the 14 IPs, extracted from the RXTE data are presented in Figs. 3-5.

For the fitting of the observed spectra we used results of our model calculations described in the previous section with, in addition, one or two (the latter only in the case of the very bright IPs GK Per and V1223 Sgr) partial covering components. We have tabulated the spectra of 51 models with WD masses ranging from 0.3 to $1.3 M_{\odot}$ at intervals of $0.02 M_{\odot}$ and 
Table 1. Summary of the RXTE observations used in this paper. PCA count rates represent count rates only from the upper layer of the PCU2 detector. HEXTE count rates are the count rates from one (of the two) clusters of detectors.

\begin{tabular}{lcrc}
\hline \hline Source & $\begin{array}{c}\text { Exp. } \\
\text { ks }\end{array}$ & $\begin{array}{c}\text { PCA }(3-20 \mathrm{keV}) \\
\text { count s }^{-1}\end{array}$ & $\begin{array}{r}\text { HEXTE }(18-60 \mathrm{keV}) \\
\text { count s }\end{array}$ \\
\hline V1223 Sgr & 90 & $13.31 \pm 0.01$ & $0.88 \pm 0.03$ \\
FO Aqr & 85 & $5.6 \pm 0.01$ & $0.27 \pm 0.03$ \\
EX Hya & 169 & $10.21 \pm 0.01$ & $0.24 \pm 0.02$ \\
AO Psc & 54 & $6.11 \pm 0.01$ & $0.12 \pm 0.03$ \\
TVCol & 86 & $6.50 \pm 0.01$ & $0.31 \pm 0.02$ \\
GK Per & 22 & $32.10 \pm 0.04$ & $3.70 \pm 0.04$ \\
V709 Cas & 36 & $7.40 \pm 0.02$ & $0.41 \pm 0.04$ \\
PQ Gem & 67 & $2.94 \pm 0.01$ & $0.10 \pm 0.03$ \\
V2400 Oph & 221 & $12.43 \pm 0.01$ & $0.30 \pm 0.01$ \\
BG CMi & 111 & $2.87 \pm 0.01$ & $0.16 \pm 0.02$ \\
V405 Aur & 26 & $2.99 \pm 0.01$ & $0.20 \pm 0.04$ \\
V1062 Tau & 35 & $3.52 \pm 0.01$ & $0.26 \pm 0.03$ \\
DO Dra & 28 & $35.78 \pm 0.01$ & $0.71 \pm 0.04$ \\
TX Col & 127 & $1.62 \pm 0.01$ & $0.08 \pm 0.02$ \\
\hline
\end{tabular}

included them in the XSPEC spectral fitting package (Arnaud 1996) for the subsequent fitting of observed spectra of IPs. In contrast to the model used by Cropper et al. (1998) our model lacks the reflected component. However, as has been shown by Cropper et al. (1998), taking into account the reflection changes the best fit white dwarf mass only slightly. On the other hand, the inclusion of spectral information at energies higher than $\sim 20 \mathrm{keV}$ helps us to further reduce the influence of the reflected component on the shape of the spectral cutoff.

The best fit parameters resulting from our models are presented in Table 2. Using the observed and absorption-corrected fluxes of the IPs we estimated their luminosities and mass accretion rates. The best fit model is shown in Figs. 3-5 by a solid line.

We compare the resulting estimates of the white dwarf masses with those measured by other methods. In the catalogue of Cataclysmic Variables (Ritter \& Kolb 2003) there are only 5 intermediate polars that have a spectroscopically measured mass. In Fig. 6 we present a comparison of our mass estimates with those given in the catalogue. It is seen that we have a relatively good agreement, however the error bars of all these estimates are quite large. The only system in which the WD likely has a higher mass than we have obtained from our X-ray spectral modelling is GK Per (during outburst). One possible explanation of this fact could be the WD's fast rotation $\left(P_{\mathrm{sp}}=351 s=5.85 \mathrm{~min}\right)$.

In our model we have implicitly assumed that the matter freely falls onto white dwarf surface from infinity. But, in reality, the matter falls freely only from the magnetospheric radius $R_{\mathrm{A}}$ (Warner 1995):

$$
\frac{R_{\mathrm{A}}}{R_{\mathrm{wd}}} \approx 2.3\left(\frac{\dot{M}}{10^{20} \mathrm{~g} / \mathrm{s}}\right)^{-2 / 7}\left(\frac{M_{\mathrm{wd}}}{M_{\odot}}\right)^{-1 / 7}\left(\frac{R_{\mathrm{wd}}}{10^{9} \mathrm{~cm}}\right)^{5 / 7}\left(\frac{B}{10^{6} \mathrm{G}}\right)^{4 / 7}
$$

The freefall velocity on the surface of the WD (at $r=R_{\mathrm{wd}}$ ) can be written as

$v_{\mathrm{ff}}^{2}\left(R_{\mathrm{A}}\right)=v_{\mathrm{ff}}^{2}(\infty)\left(1-\frac{R_{\mathrm{wd}}}{R_{\mathrm{A}}}\right)$,

which shows that the white dwarf mass estimate will depend on the ratio $R_{\mathrm{A}} / R_{\mathrm{wd}}$ (because $M_{\mathrm{wd}} \sim T_{0} \sim v_{\mathrm{ff}}^{2}$, see Eqs. (10) and (13)):

$M_{\mathrm{wd}}\left(R_{\mathrm{A}}\right)=M_{\mathrm{wd}}(\infty)\left(1-\frac{R_{\mathrm{wd}}}{R_{\mathrm{A}}}\right)$.

From this we can see that this effect is important if $R_{\mathrm{A}} / R_{\mathrm{wd}}<10$.

It is well known (e.g. Warner 1995) that accretion onto a magnetized white dwarf is only possible if $R_{\mathrm{A}}<R_{\Omega}$, where $R_{\Omega}$ is the corotation radius, i.e. the radius at which the Keplerian angular velocity equals the white dwarf rotation angular velocity). Now, for GK Per the ratio

$\frac{R_{\Omega}}{R_{\mathrm{wd}}}=2.3 \cdot P_{\mathrm{sp}}^{2 / 3}(\min )\left(\frac{M_{\mathrm{wd}}}{M_{\odot}}\right)^{1 / 3}\left(\frac{R_{\mathrm{wd}}}{10^{9} \mathrm{~cm}}\right)^{-1}$

is about 10 (assuming $M_{\mathrm{wd}}=0.8 M_{\odot}$ ). Therefore, in the case of GK Per $R_{\mathrm{A}} / R_{\mathrm{wd}}<10$ even during quiescence. During an outburst the mass accretion rate in GK Per is larger by about a factor of 10-20 (Ishida et al. 1992). Therefore, in outburst the ratio $R_{\mathrm{A}} / R_{\mathrm{wd}}$ is smaller by a factor of $\sim 2$ (see (17)). Because the spectra of GK Per were taken during an outburst, we find that $R_{\mathrm{A}} / R_{\mathrm{wd}}<5$, and we thus underestimate the mass of the white dwarf in GK Per by at least $20 \%$.

Among our targets are also other IPs in which the white dwarf rotates relatively fast. These are: V709 Cas $\left(P_{\mathrm{sp}}=\right.$ $5.12 \mathrm{~min})$, DO Dra $\left(P_{\mathrm{sp}}=8.83 \mathrm{~min}\right)$, and V405 Aur $\left(P_{\mathrm{sp}}=\right.$ $9.09 \mathrm{~min})$. Because in all these cases the ratio $R_{\Omega} / R_{\mathrm{wd}}>10$ and because the spectra in question have been taken at minimum (see Table 2 ), i.e. when $R_{\mathrm{A}} \approx R_{\Omega}$, the degree by which we underestimate the white dwarf masses in these cases is much smaller than in the case of GK Per and our mass estimates should be more accurate.

Note that our mass estimates are in better agreement with those obtained from optical methods than those obtained by Cropper et al. (1999) from their analysis of GINGA/LAC X-ray spectra ( 2-20 keV) (see Table 3 ). The masses obtained by Ramsay (2000) from his analysis of RXTE/PCA ( 2-20 keV) spectra are closer to our estimates, but also slightly higher. A possible reason for this difference could be the lack of the hard $\mathrm{X}$-ray part in the observed spectrum. We have also fitted our models to the PCA data only (see Table 3 ). We thereby obtained results which are very close to those obtained by Ramsay (2000). We have also fitted the PCA data with constant gravity models and obtained results which are practically identical to those resulting from our models with variable gravity (see also Table 3).

It is interesting to note that the parameters listed in Table 2 show a correlation of the mass accretion rate with the strength of photoabsorption. That is, in principle, anticipated for IPs because systems with higher mass accretion rates have higher $N_{\mathrm{H}}$ values. A similar effect was observed by Ishida et al. (1992) in the case of GK Per (quiescence and outburst spectra). 

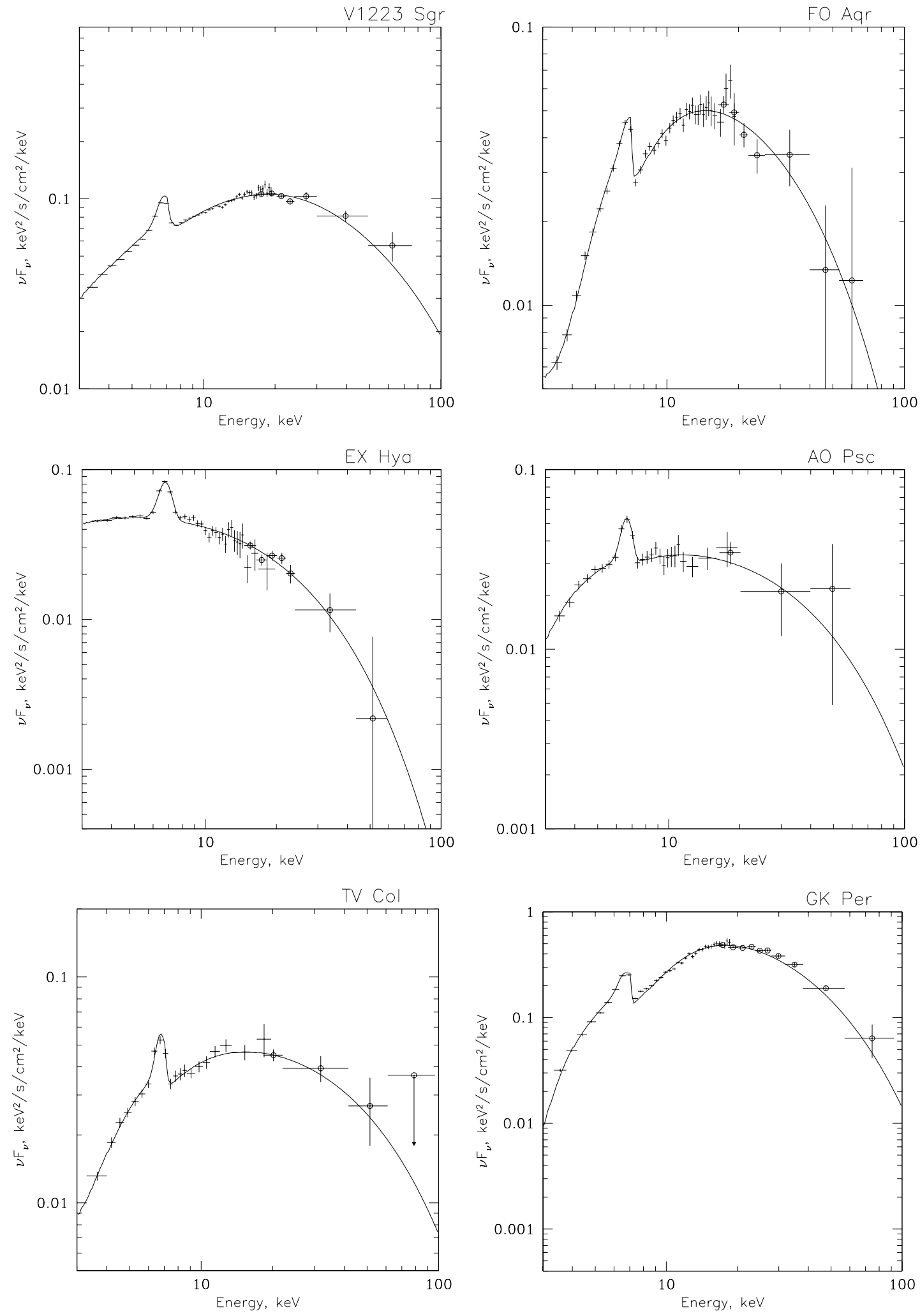

Fig. 3. Spectra of intermediate polars obtained with the RXTE observatory. Crosses denote PCA data, open circles - HEXTE data. The solid lines show the best fit model with parameters presented in Table 1. 

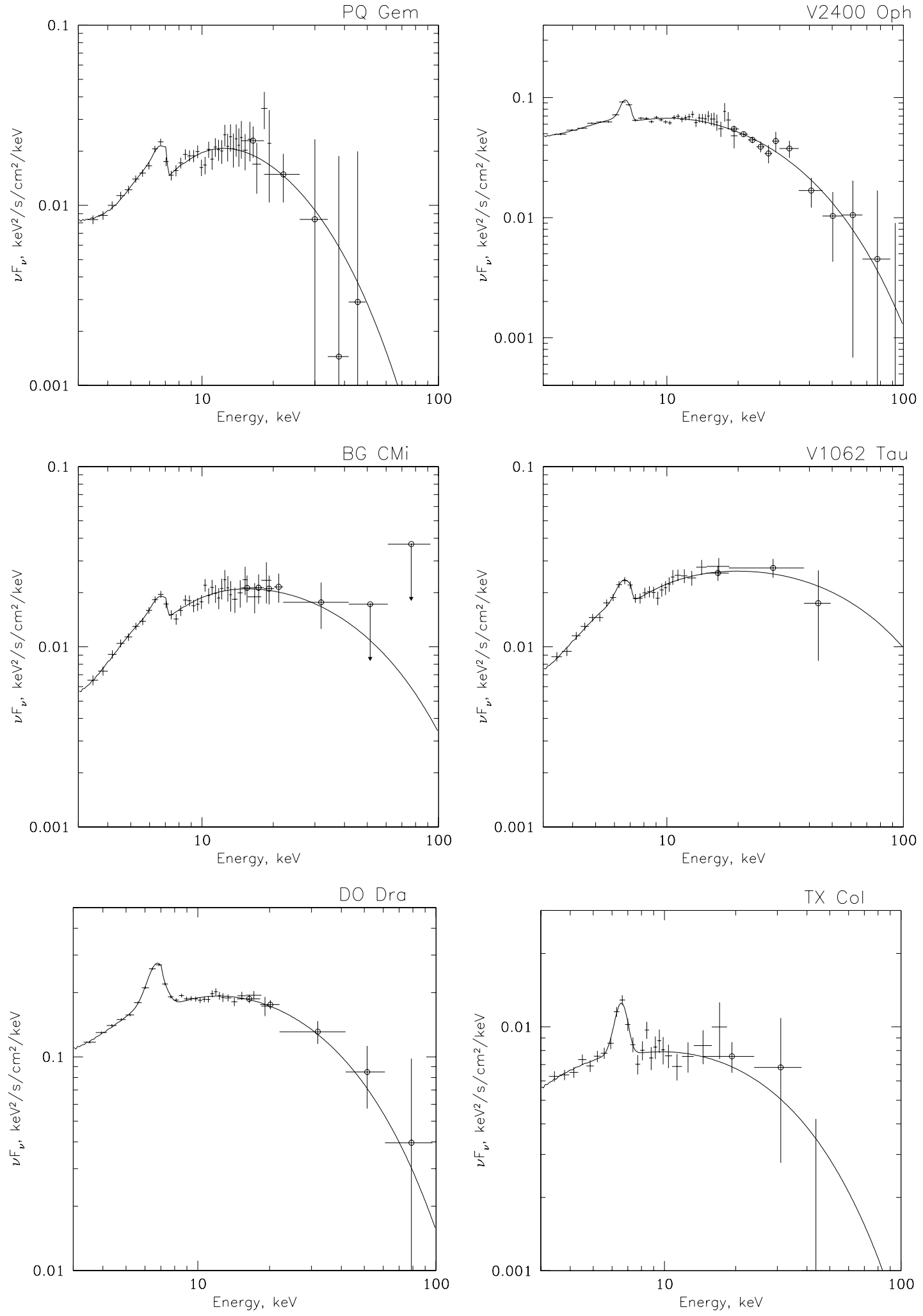

Fig. 4. The same as Fig. 3. 

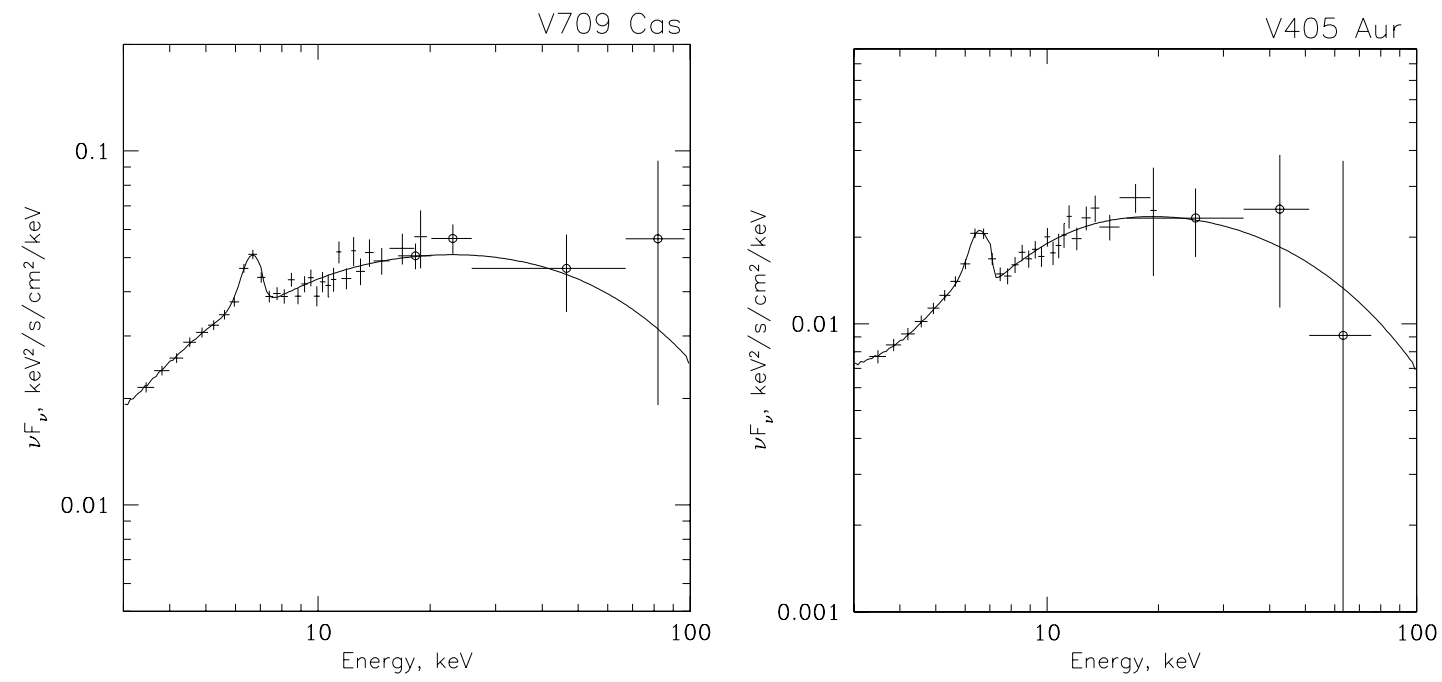

Fig. 5. The same as Fig. 3.

Table 2. Best fit model parameters and inferred physical system parameters. Here $C_{F}$ is the partial covering coefficient, $F_{3-100}$ the observed $\mathrm{X}$-ray flux in the 3-100 keV band, $F_{0.1-100}$ the unabsorbed X-ray flux in the $0.1-100 \mathrm{keV}$ band, both in $10^{-11} \mathrm{erg} \mathrm{cm}^{-2} \mathrm{~s}^{-1}$ units. The distances for V405 Aur, V709 Cas, PQ Gem and V2400 Oph are assumed.

\begin{tabular}{|c|c|c|c|c|c|c|c|c|c|}
\hline Name & $\begin{array}{l}M_{\mathrm{wd}}, \\
M_{\odot}\end{array}$ & $C_{F}$ & $\begin{array}{l}N_{\mathrm{H}}, \\
10^{22} \mathrm{~cm}^{-2}\end{array}$ & $F_{3-100}$ & $F_{0.1-100}$ & $\begin{array}{l}\dot{M} \\
10^{16} \mathrm{~g} \mathrm{~s}^{-1}\end{array}$ & $\begin{array}{l}L_{\mathrm{x}}, 10^{33} \\
\mathrm{erg} \mathrm{s}^{-1}\end{array}$ & $\begin{array}{l}d \\
\mathrm{pc}\end{array}$ & $\chi^{2}$ (d.o.f.) \\
\hline \multirow[t]{2}{*}{ V1223 Sgr } & $0.95 \pm 0.05$ & $1.0^{a}$ & $3.7 \pm 1$ & 4.2 & 7.3 & 12.6 & 24.3 & $527^{1}$ & $105.4(63)$ \\
\hline & & $0.57 \pm 0.05$ & $28 \pm 3$ & & & & & & \\
\hline FO Aqr & $0.60 \pm 0.05$ & $0.9 \pm 0.1$ & $36 \pm 4$ & 1.5 & 4.9 & 10.2 & 9.4 & $400^{2}$ & $62.1(65)$ \\
\hline EX Hya & $0.50 \pm 0.05$ & 1.0 & $0.6 \pm 1.0$ & 1.5 & 3.4 & 0.25 & 0.17 & $64.5^{3}$ & $85.3(65)$ \\
\hline AO Psc & $0.65 \pm 0.05$ & $0.95 \pm 0.1$ & $10 \pm 5$ & 1.3 & 2.5 & 4.7 & 4.9 & $420^{4}$ & $55.8(65)$ \\
\hline TVCol & $0.84 \pm 0.06$ & $0.81 \pm 0.3$ & $21 \pm 5$ & 1.7 & 3.5 & 3.4 & 5.7 & $368^{5}$ & $63.4(65)$ \\
\hline \multirow[t]{2}{*}{ GK Per } & $0.59 \pm 0.05$ & $1.0^{a}$ & $20 \pm 2$ & 12.5 & 88.6 & 81.5 & 156 & $340^{6}$ & $110.5(63)$ \\
\hline & & $0.75 \pm 0.05$ & $125 \pm 10$ & & & & & & \\
\hline V709 Cas & $0.90 \pm 0.10$ & $0.5 \pm 0.1$ & $29 \pm 5$ & 2.3 & 3.7 & 5.76 & 11.1 & (500) & $56.3(65)$ \\
\hline PQ Gem & $0.65 \pm 0.2$ & 1.0 & $5 \pm 1$ & 0.80 & 1.3 & 3.7 & 3.9 & (500) & $38.7(65)$ \\
\hline V2400 Oph & $0.59 \pm 0.05$ & $0.4 \pm 0.1$ & $29 \pm 10$ & 2.43 & 5.6 & 18.7 & 16.75 & (500) & $78.5(65)$ \\
\hline BG CMi & $0.85 \pm 0.12$ & $0.70 \pm 0.05$ & $25 \pm 6$ & 0.77 & 1.54 & 5.3 & 9.0 & $700^{7}$ & $57.4(65)$ \\
\hline V405 Aur & $0.90 \pm 0.10$ & $0.6 \pm 0.1$ & $39 \pm 3$ & 0.9 & 1.7 & 2.65 & 5.09 & (500) & $51.6(65)$ \\
\hline V1062 Tau & $1.00 \pm 0.20$ & $0.6 \pm 0.1$ & $21 \pm 7$ & 1.1 & 1.8 & 2.2 & 5.4 & $500^{8}$ & $45.6(65)$ \\
\hline DO Dra & $0.75 \pm 0.05$ & $0.4 \pm 0.1$ & $20 \pm 5$ & 7.8 & 14.4 & 3.1 & 4.1 & $155^{9}$ & $69.7(65)$ \\
\hline TX Col & $0.70 \pm 0.30$ & $<0.4$ & $<60$ & 0.32 & 0.58 & 1.8 & 2.1 & $550^{10}$ & $52.6(65)$ \\
\hline
\end{tabular}

${ }^{a}$ - For the systems V1223 Sgr and GK Per which have very high mass accretion rates we used two absorbers.

References to distance estimates: 1. Beuermann et al. (2004); 2. McHardy et al. (1987); 3. Beuermann et al. (2003); 4. Hellier et al. (1991); 5. McArthur et al. (2001); 6. Warner (1987); 7. Berriman (1987); 8. Szkody \& Silber (1996); 9. Mateo et al. (1991); 10. Buckley \& Tuohy (1989).

\section{Conclusions}

Determining the mass of the white dwarf in cataclysmic variables has been, and still is, a tricky business. Because the number of known double-lined eclipsing systems which have their radial velocities reliably measured and for which mass determinations are straightforward is still very small (see e.g. Ritter \& Kolb 2003), the majority of mass determinations which have been published are based on additional and often not wellfounded assumptions. Consequently, the relaibility of the resulting masses is difficult to asses. It is therefore important that magnetic cataclysmic variables offer us an additional possibility for determining the mass of the white dwarf. Accreted near the magnetic poles the matter forms a shock wave above the WD surface. The temperature and the emergent spectrum of the hot, optically thin post-shock region (PSR) depend in a first approximation on the mass of the white dwarf only. Therefore it is possible to estimate WD masses in polars and intermediate polars by fitting their hard X-ray spectra with model spectra of the PSR. However, the temperature of the PSR at the surface of a massive white dwarf is so high, i.e. (10-30 keV) that a hard X-ray spectrum emerges, the cutoff energy of which is barely within the spectral bands of the ASCA, GINGA, and RXTE/PCA spectrometers ( $h v<10-20 \mathrm{keV}$ ) (see Ramsay 2000). Therefore, to obtain reliable mass estimates for 
Table 3. Comparison of different mass estimates. We present here masses obtained from broad band (3-100 keV) spectral fitting (Col. 2) and narrow band spectral fitting - our model (Cols. 3 and 4), work of Ramsay 2000 (Col. 5), work of Cropper et al. 1999 (Col. 6), and work of Ezucka \& Ishida 1999 (Col. 7).

\begin{tabular}{|c|c|c|c|c|c|c|}
\hline \multirow[t]{2}{*}{ Name } & $R X T E^{1}$ & $\mathrm{PCA}^{2}$ & $\mathrm{PCA}^{3}$ & $\mathrm{PCA}^{4}$ & $G I N G A^{5}$ & $A S C A^{6}$ \\
\hline & $M_{\odot}$ & $M_{\odot}$ & $M_{\odot}$ & $M_{\odot}$ & $M_{\odot}$ & \\
\hline V1223 Sgr & $0.95 \pm 0.05$ & $1.25 \pm 0.05$ & $1.25 \pm 0.05$ & 1.1 & & 1.28 \\
\hline FO Aqr & $0.60 \pm 0.05$ & $0.82 \pm 0.07$ & $0.83 \pm 0.07$ & 0.88 & 0.92 & 1.05 \\
\hline EX Hya & $0.50 \pm 0.05$ & $0.44 \pm 0.02$ & $0.45 \pm 0.02$ & 0.45 & 0.46 & 0.48 \\
\hline AO Psc & $0.65 \pm 0.05$ & $0.56 \pm 0.02$ & $0.57 \pm 0.02$ & 0.60 & 0.56 & 0.40 \\
\hline TV Col & $0.84 \pm 0.06$ & $0.95 \pm 0.03$ & $0.97 \pm 0.03$ & 0.96 & 1.30 & 0.51 \\
\hline GK Per & $0.59 \pm 0.05$ & $1.24 \pm 0.10$ & $1.23 \pm 0.15$ & & & 0.52 \\
\hline V709 Cas & $0.90 \pm 0.10$ & $1.20 \pm 0.10$ & $1.18 \pm 0.10$ & 1.08 & & \\
\hline PQ Gem & $0.65 \pm 0.20$ & $1.10 \pm 0.20$ & $1.13 \pm 0.20$ & & 1.29 & \\
\hline V2400 Oph & $0.59 \pm 0.05$ & $0.68 \pm 0.05$ & $0.68 \pm 0.05$ & 0.71 & & 0.68 \\
\hline BG CMi & $0.85 \pm 0.12$ & $0.90 \pm 0.10$ & $0.90 \pm 0.10$ & 1.20 & 1.09 & \\
\hline V405 Aur & $0.90 \pm 0.10$ & $1.18 \pm 0.10$ & $1.17 \pm 0.10$ & 1.10 & & $>0.54$ \\
\hline V1062 Tau & $1.00 \pm 0.20$ & $1.27 \pm 0.10$ & $1.28 \pm 0.10$ & 0.90 & & \\
\hline DO Dra & $0.75 \pm 0.05$ & $0.86 \pm 0.05$ & $0.84 \pm 0.05$ & & & \\
\hline TX Col & $0.70 \pm 0.30$ & $0.90 \pm 0.20$ & $0.91 \pm 0.20$ & 0.73 & 0.48 & 0.66 \\
\hline
\end{tabular}

References to the WD mass estimates: 1. PCA+HEXTE, this work; 2. PCA only, this work; 3. PCA only, constant gravity model; 4. Ramsay (2000); 5. Cropper et al.( 1999); 6. Ezucka and Ishida (1999).

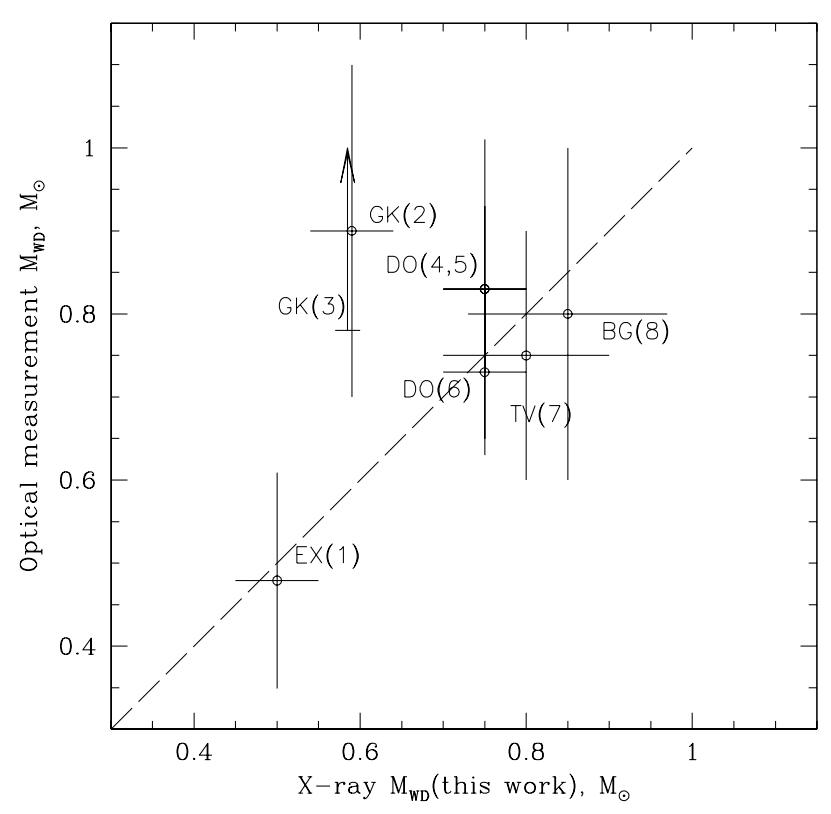

Fig. 6. Comparison of the masses of the white dwarfs as estimated from RXTE broadband spectra (this work) with estimates obtained by other methods. (1) - Beuermann et al. (2003); (2) - Crampton et al. (1986); (3) - Reinsch (1994); (4) - Haswell et al. (1997); (5) - Mateo et al. (1991); (6) - Friend et al. (1990); (7) - Hellier (1993); (8) Penning (1985).

the white dwarf in IPs it is necessary to take into account the flux distribution of the hard X-rays emerging from the PSR.

In this paper we present the WD masses of 14 IPs estimated from the fitting of broad band (3-100 keV) $R X T E / \mathrm{PCA} / \mathrm{HEXTE} \mathrm{X}$-ray spectra with theoretical spectra of the PSR calculated by us. We have not taken into account cyclotron cooling in the PSR and, therefore, did not consider polars, where it can be important. Our estimates give lower WD masses than those obtained from GINGA/LAC (Cropper et al. 1999 ) and RXTE/PCA (Ramsay 2000) data. We also find satisfactory agreement between our WD mass estimates and those obtained by means of optical spectroscopic methods. In conclusion, this shows that it is absolutely necessary to use the hard $\mathrm{X}$-ray data $(E>20 \mathrm{keV})$ to obtain reliable WD mass estimates in mCVs.

Acknowledgements. This work was supported by the DAAD Fellowship, Academy of Finland (grant 100488), the Jenny and Antti Wihuri Foundation, Russian Foundation of Fundamental Research (grant 02-02-17174) and by the President's program for support of leading science schools (grant NSh - 1789.2003.2).

\section{References}

Aizu, K. 1973, Prog. Theor. Phys., 49, 1184

Arnaud, K. A. 1996, in Astronomical Data Analysis Software and Systems V, ed. G. Jacoby, \& J. Barnes (San Francisco: Astron. Soc. Pac.), ASP Conf. Ser., 101, 10

Beardmore, A., Osborne, J., \& Hellier, C. 2000, MNRAS, 315, 307

Belle, K., Howell, S., Sion, E., Long, K., \& Szkody, P. 2003, ApJ, 587, 373

Berriman, G. 1987, A\&A, 412, 821

Beuermann, K., Harrison, Th., McArthur, B., Benedict, G., \& Gänsicke, B. 2003, A\&A, 412, 821

Beuermann, K., Harrison, Th., McArthur, B., Benedict, G., \& Gänsicke, B. 2004, A\&A, 419, 291

Buckley, D. A. H., \& Tuohy, I. R. 1989, ApJ, 344, 376

Crampton, D., Cowley, A. P., \& Fisher, W. A. 1986, ApJ, 300, 788 
Cropper, M., Ramsay, G., \& Wu, K. 1998, MNRAS, 293, 222

Cropper, M., Wu, K., Ramsay, G., \& Kocabiyik, A. 1999, MNRAS, 306,684

Done, C., \& Magdziarz, P. 1998, MNRAS, 298, 737

Ezuka, H., \& Ishida, M. 1999, ApJS, 120, 277

Frank, J., King, A., \& Raine, D. 2002, Accretion Power in Astrophysics (Cambridge: Cambridge Univ. Press), 3rd edition

Friend, M. T., Martin, J. S., Smith, R. C., \& Jones, D. H. P. 1990, MNRAS, 246, 637

Haswell, C. A., Patterson, J., Thorstensen, J. R., Hellier, C., \& Skillman, D. R. 1997, ApJ, 476, 847

Hellier, C., Cropper, M., \& Mason, K. 1991, MNRAS, 248, 233

Hellier, C. 1993, MNRAS, 264, 132

Ishida, M. 1991, Ph.D. Thesis, Univ. of Tokio

Ishida, M., Sakao, T., Makishima, K., et al. 1992, MNRAS, 254, 647

Lamb, D., \& Masters, A. 1979, ApJ, 234, L117

Mateo, M., Szcody, P., \& Garnavich, P. 1991, ApJ, 370, 370

McArthur, B. E., Benedict, G. F., Lee, J., et al. 2001, ApJ, 560, 907

McHardy, I. M., Pye, J. P., Fairall, A., \& Menzies, J. 1987, MNRAS, 225,355

Mewe, R., Kaastra, D. S., \& Liedahl, D. A. 1995, Legacy (Journal of HEASARC), 6, 16
Nauenberg, M. 1972, ApJ, 175, 417

Penning, W. R. 1985, ApJ, 289, 300

Ramsay, G. 2000, MNRAS, 314, 403

Reinsch, K. 1994, A\&A, 281, 108

Ritter, H., \& Kolb, U. 2003, A\&AS, 404, 301

Rothschild, R. E., Gruber, D. E., Knight, F. K., et al. 1981, ApJ, 250, 723

Rothschild, R. E., Blanco, P. R., Gruber, D. E., et al. 1998, ApJ, 496, 538

Schwope, A. D., Hambaryan, V., Schwarz, R., Kanbach, G., \& Gänsicke, B. T. 2002, A\&A, 392, 541

Szkody, P., \& Silber, A. 1996, AJ, 112, 289

Sutherland, R. S., \& Dopita, M. A. 1993, ApJS, 88, 253

Warner, B. 1987, MNRAS, 227, 23

Warner, B. 1995, Cataclysmic variable stars (Cambridge: Cambridge Univ. Press)

Woelk, U., \& Beuermann, K. 1996, A\&A, 306, 232

Wu, K., Chanmugam, G., \& Shaviv, G. 1994, ApJ, 426, 664

Wu, K. 2000, Space Sci. Rev., 93, 611

Zombeck, M. V. 1990, Handbook of Astronomy and Astrophysics (Cambridge: Cambridge Univ. Press) 